\title{
Toward dual-wavelength functional photoacoustic endoscopy: laser and peripheral optical systems development
}

Joon-Mo Yang, Christopher Favazza, Ruimin Chen, Junjie Yao, Xin Cai, et al.

Joon-Mo Yang, Christopher Favazza, Ruimin Chen, Junjie Yao, Xin Cai, Konstantin Maslov, Qifa Zhou, K. Kirk Shung, Lihong V. Wang, "Toward dual-wavelength functional photoacoustic endoscopy: laser and peripheral optical systems development," Proc. SPIE 8223, Photons Plus Ultrasound: Imaging and Sensing 2012, 822316 (9 February 2012); doi: $10.1117 / 12.909163$

SPIE. Event: SPIE BiOS, 2012, San Francisco, California, United States 


\title{
Toward dual-wavelength functional photoacoustic endoscopy: laser and peripheral optical systems development
}

\author{
Joon-Mo Yang ${ }^{1}$, Christopher Favazza ${ }^{1}$, Ruimin Chen ${ }^{2}$, Junjie Yao ${ }^{1}$, Xin Cai ${ }^{1}$, Konstantin Maslov ${ }^{1}$ \\ Qifa Zhou ${ }^{2}$, K. Kirk Shung ${ }^{2}$, and Lihong V. Wang ${ }^{1 *}$ \\ 'Optical Imaging Laboratory, Department of Biomedical Engineering, Washington University in St. \\ Louis, One Brookings Drive, Campus Box 1097, St. Louis, MO 63130, USA \\ ${ }^{2}$ Ultrasonic Transducer Resource Center, Department of Biomedical Engineering, University of \\ Southern California, 1042 Downey Way, University Park, DRB 130, Los Angeles, CA 90089, USA
}

\begin{abstract}
Photoacoustic endoscopy (PAE) provides unique functional information with high spatial resolution at super depths. The provision of functional information is predicated on its strong spectroscopic imaging ability, and its deep imaging capability is derived from its ultrasonic detection of diffused photon absorption. To accurately image functional physiological parameters, it is necessary to rapidly alternate laser pulses of sufficient energy and different wavelengths. In this study, we developed peripheral optical systems for PAE based on two identical pulsed-laser systems to achieve the fast laser wavelength switching that is essential for accurate functional imaging. Each laser system was comprised of a tunable dye laser pumped by a solid-state, diode-pumped Nd:YLF laser. Both systems deliver adequate energy at the scanning head of the endoscope for imaging biological tissue in the optically diffusive regime $(\sim 0.3-0.6 \mathrm{~mJ}$ per pulse with a repetition rate of $\sim 1 \mathrm{kHz}$ ). In this paper, we introduce the employed laser systems and design of the light delivery optics, and present results from an ex vivo animal imaging experiment that validates the system's multi-wavelength functional imaging capability.
\end{abstract}

Keywords: Photoacoustic endoscopy, functional imaging, photoacoustic tomography, spectroscopy, gastrointestinal endoscopy.

\section{INTRODUCTION}

Photoacoustic endoscopy (PAE) ${ }^{1-6}$ holds promise for clinical application because it provides high-resolution and functional, optical contrast at super depths that far exceeds the penetration limits of other optical endoscopic modalities, such as endoscopic optical coherence tomography $(\mathrm{OCT})^{7-9}$, confocal endoscopy ${ }^{10}$, or endoscopic polarized scanning spectroscopy ${ }^{11,12}$. Deep imaging is made possible by interrogating the target with diffused photons, which can penetrate up to several centimeters into soft tissue. Absorption of either diffuse or ballistic photons will indiscriminately induce acoustic waves, i.e., photoacoustic (PA) waves, through thermoelastic expansion, and these waves will be subsequently detected by an ultrasonic (US) transducer ${ }^{1,13}$. Hence, ultrasound resolution optical contrast images can be produced from objects located in an optically diffusive regime. This technique recently has gained much interest ${ }^{1,5,13-15}$ because it can uniquely provide optical absorption contrast, rich with spectroscopic information, at clinically relevant depths. By simply changing the excitation laser wavelength, physiologically-specific, optical absorption-based contrast can be acquired from various endogenous contrast agents, enabling quantitative assessment of functional information (e.g., $\mathrm{Hb}$ concentration, $\left.\mathrm{sO}_{2}\right)^{13,16-21}$.

Multiscale imaging is another key feature of PA imaging ${ }^{13,14,22}$, as demonstrated in many studies ${ }^{23-29}$. By choosing optical illumination and acoustic detection parameters appropriately, one can produce tomographic images whose resolution ranges from sub-micron at shallow depths ${ }^{25}$ to $\sim$ millimeter resolution at depths greater than several centimeters $^{26-29}$. Our current interest is to develop an endoscopic imaging system targeting deep tissue. To realize this goal, a laser system with sufficient pulse energy is required to optically excite targets in the diffusive regime. Also,

\footnotetext{
* Corresponding author: lhwang@biomed.wustl.edu
}

Photons Plus Ultrasound: Imaging and Sensing 2012, edited by Alexander A. Oraevsky, Lihong V. Wang, Proc. of SPIE Vol. 8223, 822316 - (c) 2012 SPIE · CCC code: 1605-7422/12/\$18 · doi: 10.1117/12.909163 
multiple laser wavelengths are required to extract functional information. Furthermore, the laser pulse repetition rate must be high for acceptable in vivo imaging and accurate measurement of dynamic physiological parameters. To satisfy this requirement, we employed two identical pulsed laser systems that provided large pulse energies at high repetition rates. Also, we developed peripheral optical systems to deliver the laser beams efficiently. Lastly, using an endoscopic probe that we developed recently ${ }^{6}$, we demonstrated the system's multi-wavelength imaging capability through an ex vivo animal experiment.

\section{MATERIALS AND METHODS}

\subsection{Photoacoustic endoscopic probe}

We have created and continue to develop endoscopic probes and associated systems for gastrointestinal tract imaging applications ${ }^{2-4,6}$. As shown in Figure 1, the main feature of our recently developed endoscopic probe is its built-in scanning mechanism that employs a scanning mirror and micromotor. A multimode optical fiber (OF1, 0.22 NA, 365 $\mu \mathrm{m}$ core diameter, BFL22-365, Thorlabs) couples and guides laser pulses from the engaged laser systems and emits the laser pulses through a central hole in the US transducer. After exiting the fiber, the laser beams are then directed by a reflector (or scanning mirror) towards the sample, exciting the target and generating PA waves. Some of the generated PA waves are collected by the probe and propagate to the scanning mirror, which reflects the PA waves to the US transducer, which then converts them into electric signals. The scanning mirror, driven by a geared micromotor (gear ratio, 254:1; Namiki Precision), reflects both the laser light and acoustic waves, and performs rotational scanning for cross-sectional (B-scan) imaging at a frame rate of $\sim 4 \mathrm{~Hz}$. The detected PA signals are amplified by a US pulserreceiver (5072 PR, Panametrics-NDT, Olympus), digitized (NI PCI-5124, National Instruments) and recorded by a computer. The scanning mirror steers both the laser beam and the PA waves, and it enables static mounting of the associated illumination and US detection units. Importantly, the scanning mirror inserts no additional propagation losses into the US detection because it exhibits total internal acoustic reflection within the acceptance angles of the US transducer.

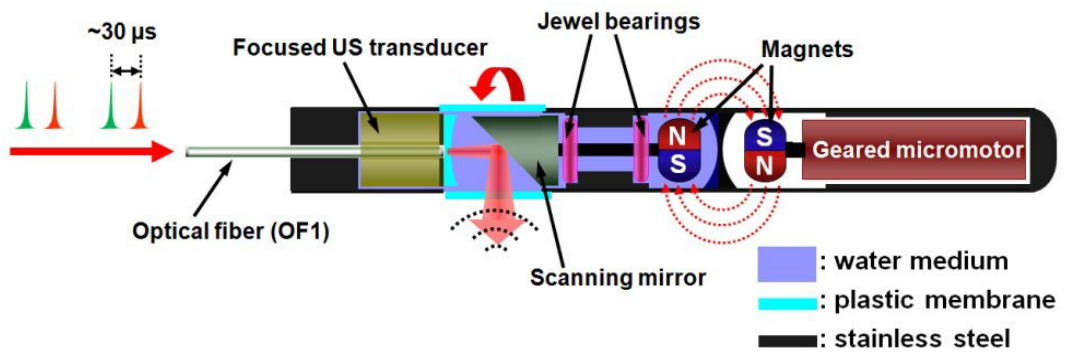

Figure 1. Schematic of the photoacoustic endoscopic probe

To provide acoustic impedance matching, the endoscope's inner space is filled with de-ionized water and sealed with an optically and acoustically transparent, $\sim 40 \mu \mathrm{m}$ thick plastic membrane. However, the micromotor is physically isolated from the liquid medium to provide an in-air working environment. The torque required to rotate the mirror is transferred through magnetic coupling of the mirror and the micromotor. All optical, US, and mechanical elements are encapsulated in a streamlined stainless steel tube that allows smooth intracavitary movement. The optical fiber (OF1), the US transducer's signal wire, and the micromotor's wires are also catheterized in a flexible polyethylene terephthalate (PET) plastic tube $\sim 1 \mathrm{~m}$ long. For spectroscopic imaging, multiple laser beams of different wavelengths (dual-wavelengths in this study) can be coupled to the proximal end of the light guiding optical fiber (OF1). More information on the endoscope's structure is available in our previous reports ${ }^{2-4}$.

\subsection{Laser system and peripheral optics}

For dual-wavelength imaging, we employ two identical pulsed laser systems. In our laboratory, the laser systems happened to be physically separated by a large distance $(\sim 10 \mathrm{~m})$. Each pulsed laser system is comprised of a tunable dye laser (Cobra HRR, Sirah) pumped by a solid-state, diode-pumped Nd:YLF laser (523 nm, INNOSLAB IS811-E, EdgeWave) and is triggered by an angular position-encoded TTL signal provided by the micromotor's driver circuit. At 
every $1.42^{\circ}$ rotation of the scanning mirror, both the first and second laser beams are independently fired from the two laser systems with a constant time delay of $\sim 30 \mu \mathrm{s}$, and the corresponding PA A-line signals are detected by the US transducer (Fig. 1). The 30- $\mu$ s time delay is necessary to avoid signal overlap. However, this delay does not significantly affect co-registration of the images because the time interval is much shorter than the scanning mirror's rotation speed $(\sim 4 \mathrm{~Hz})$. This imaging scheme enables rapid dual-wavelength PA imaging and co-registration.

The $\sim 10 \mathrm{~m}$ distance between the two laser systems necessitated developing a light delivery system. To deliver the two laser beams efficiently, we created light guiding and coupling optics as shown in Figure 2(a). Laser beams emitted from the first laser system (Laser set 1) were coupled to a $\sim 10$-m long multi-mode optical fiber $(\mathbf{O F 2}, 365 \mu \mathrm{m}, 0.22$ NA, BFL22-365, Thorlabs) by a $0.15 \mathrm{NA}$ aspheric lens (L0, $f=18.40 \mathrm{~mm}, \mathrm{C} 280 \mathrm{TME}-\mathrm{A}$, Thorlabs) and delivered to an optical coupler-combiner positioned near the second laser system (Laser set 2) [Fig. 2(a)]. Emitted laser beams from the optical fiber (OF2) were collimated by a lens $(\mathbf{L 1}, f=11 \mathrm{~mm}, 0.25 \mathrm{NA}, \mathrm{F} 220 \mathrm{SMA}-\mathrm{A}$, Thorlabs) and passed through a beam splitter ( $70 \%$ transmittance and 30\% reflectance, EBP1, Thorlabs). The laser beams were then focused by additional lens ( $\mathbf{L} 2, f=11 \mathrm{~mm}, 0.25 \mathrm{NA}, \mathrm{C} 220 \mathrm{TME}-\mathrm{A}$, Thorlabs) and coupled to the proximal end of the endoscopic probe's optical fiber (OF1). The endoscope's optical fiber (OF1) and the 10-m long light guiding optical fiber (OF2) had the same core diameter $(365 \mu \mathrm{m})$ and numerical aperture $(0.22)$. Also, the two lenses (L1 and L2) had the same optical specifications and were placed in a symmetric configuration [Fig. 2(b)]. The second laser beam (Laser set 2) was directly coupled to the beam combiner after free-space propagation, as shown in Figures 2(a) and 2(c). The final output energies of the first (from Laser set 1) and second (from Laser set 2) laser beams at the endoscope's scanning head were $\sim 40 \%$ and $\sim 20 \%$, respectively, relative to the input energy. The absolute energy values of the laser beams from the dye laser varied with wavelength. With the implemented laser and optical systems, we could achieve maximum output energies in the range of $0.3-0.6 \mathrm{~mJ} / \mathrm{pulse}$ at a pulse repetition rate of $1 \mathrm{kHz}$. Considering the $70 \%$ transmittance of the beam splitter, the $40 \%$ delivery of the first laser beam was quite efficient. For real endoscopic imaging experiments, we adjusted the output power of the pump laser to equalize the energies of the two laser beams to around $0.3 \mathrm{~mJ} / \mathrm{pulse}$. Depending on the imaging target of interest, we adjusted the transmittance (or reflectance) of described beam splitter [Fig. 2(a)] appropriately to more efficiently harvest the required pulse energies from both laser wavelengths.

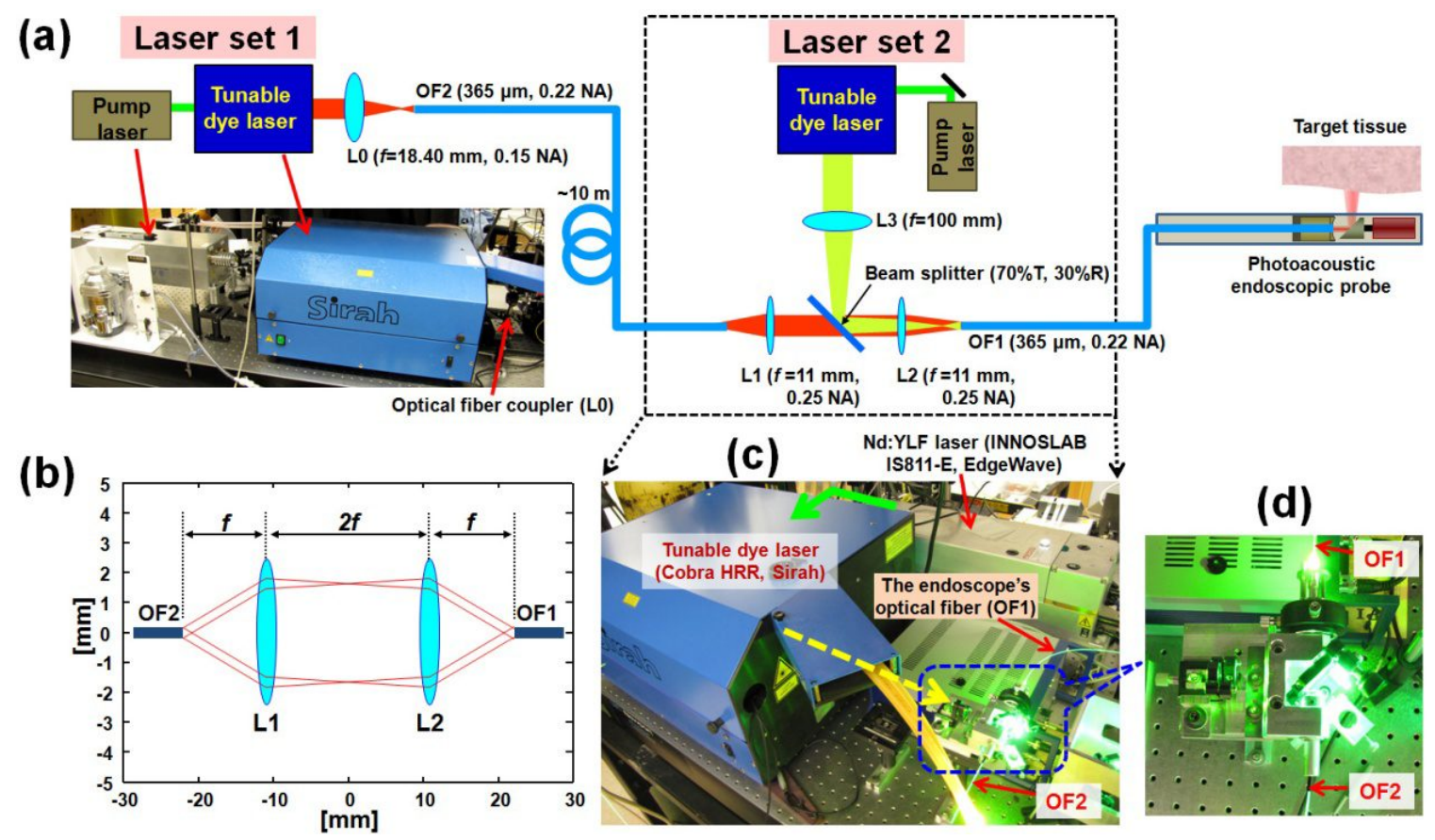

Figure 2. (a) Schematic of the laser and light delivery optical system. (b) A ray tracing diagram of the beam combiner shown in (a), including physical dimensions. $f$ represents the focal length of the lenses. The beam splitter was placed between the two lenses. (c) A photo showing the laser system (Laser set 2) and light coupling optics connected to an endoscopic probe. This photo region corresponds to the dashed rectangular area shown in the schematic (a). (d) A photo showing the beam combiner in (c). 
Since the laser beams' spot sizes were $\sim 2 \mathrm{~mm}$ at the endoscope's scanning head, the approximate laser fluence was $\sim 10$ $\mathrm{mJ} / \mathrm{cm}^{2}$. With this laser fluence and the capacity of the employed digitizer, we could achieve the endoscope's maximal radial imaging depth of $\sim 7 \mathrm{~mm}$ from the endoscope's surface. The endoscope's angular field-of-view (FOV) was limited to approximately $270^{\circ}$, due to partial blocking by the probe housing. Experimentally measured PA resolutions were $\sim 55 \mu \mathrm{m}$ in the radial direction and $\sim 80 \mu \mathrm{m}$ in the transverse direction, but the transverse resolution varied with target distance.

\subsection{Animal experiment}

To test the endoscopic system's dual-wavelength imaging capability, we performed an ex vivo imaging experiment using an adult Sprague Dawley rat ( $\sim 50$ g; Harlan National Customer Service Center). Prior to endoscopic imaging, we euthanized the animal by a pentobarbital overdose $(150 \mathrm{mg} / \mathrm{kg}$, IP) and surgically opened the abdomen [Fig. 3]. Once the animal was properly positioned, we introduced medical ultrasound gel into the descending colon via a small plastic tube and also spread gel around the colon. The ultrasound gel provided acoustic coupling between the tissue and US transducer and lubricated the probe during colon insertion through the anus. Then, we inserted the endoscopic probe into the colon $\sim 8 \mathrm{~cm}$ deep from the animal's anus and performed volumetric scans over a $\sim 8 \mathrm{~cm}$ range during constant pullback translation of the probe at a mechanical governed speed of $\sim 160 \mu \mathrm{m} / \mathrm{s}$. Each volumetric scan required a scanning time of $\sim 8.3 \mathrm{~min}$. About $2000 \mathrm{~B}$-scan slices with longitudinal spacings of $\sim 40 \mu \mathrm{m}$ were acquired for each imaging mode. In this experiment, we set the wavelengths of the laser systems to $523 \mathrm{~nm}$ (Laser set 1) and $640 \mathrm{~nm}$ (Laser set 2), respectively. The $523 \mathrm{~nm}$ laser pulses were directly coupled from the pump laser, not from the tunable dye laser, of Laser set 1. Due to the bypass of the dye laser for the $523 \mathrm{~nm}$ laser pulses and the spectral efficiency profile of the dye laser, in which the efficiency decreases with increasing wavelength, there was a great discrepancy in the maximum pulse energies at these laser wavelengths. Since the maximum $640 \mathrm{~nm}$ laser energy was generally weak and was much weaker than the $523 \mathrm{~nm}$ laser energy, we replaced the beam splitter shown in Figure 2(a) with a different one (30\% transmittance and 70\% reflectance, NT46-607, Edmund Optics) to harvest more energy from the $640 \mathrm{~nm}$ laser pulse.

All procedures in the experiment followed the protocol approved by the Institutional Animal Care and Use Committee at Washington University in St. Louis.

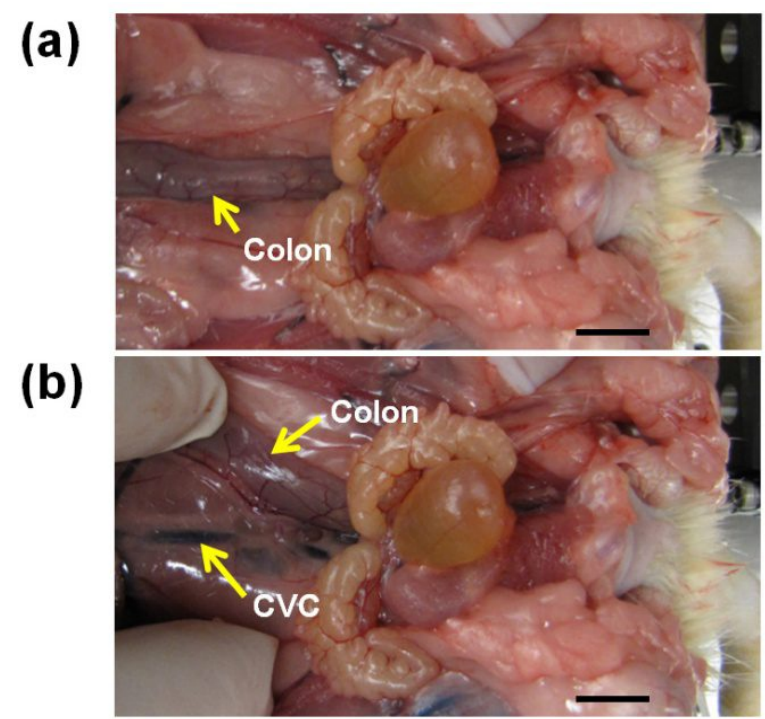

Figure 3. Photos showing the anatomic structures near the rat colon. The intact (a) and shifted (b) colon is shown. In image (b), one can see several blood vessels distributed in the colon wall and another large blood vessel, which represents the caudal vena cava (CVC), running at the back cavity wall of the animal body. Although the animal was dead, an obvious color difference between the caudal vena cava, which appeared very dark, and other blood vessels in the colon wall implied an $\mathrm{sO}_{2}$ difference between the vessels. The scale bars represent $1 \mathrm{~cm}$. 


\section{RESULTS AND DISCUSSION}

In Figure 4(a), we present a radial-maximum amplitude projection (RMAP) image that was spectrally-processed from a set of dual-wavelength $(523 \mathrm{~nm}$ and $640 \mathrm{~nm}$ ) PA volumetric data acquired from the dorsal region of a rat colon ex vivo. The imaged area covers $\sim 270^{\circ}$ angular FOV (vertical axis) and an $\sim 8 \mathrm{~cm}$ long pullback range (horizontal axis). This RMAP image shows approximate $\mathrm{sO}_{2}$ levels of the imaged blood vessels. Although the image was acquired ex vivo [Fig. 3(a)], $\mathrm{sO}_{2}$ differences are shown in several prominent blood vessels, such as the caudal vena cava (CVC) and common iliac vein (CIV), which both show lower oxygenation than other surrounding vessels.

In Figure 4(b), we present a volume-rendered image processed from the same data set displayed in Figure 4(a). This image covers an $8 \mathrm{~cm}$ long cylindrical volume with a $12 \mathrm{~mm}$ imaging diameter, and was created from PA data acquired at a $523 \mathrm{~nm}$ laser wavelength. The volume-rendered PA image shows the vasculature at the colon wall. In vivo animal imaging experimental results and discussion of the potential clinical applications of this system will be presented in our upcoming paper ${ }^{6}$.

(a)

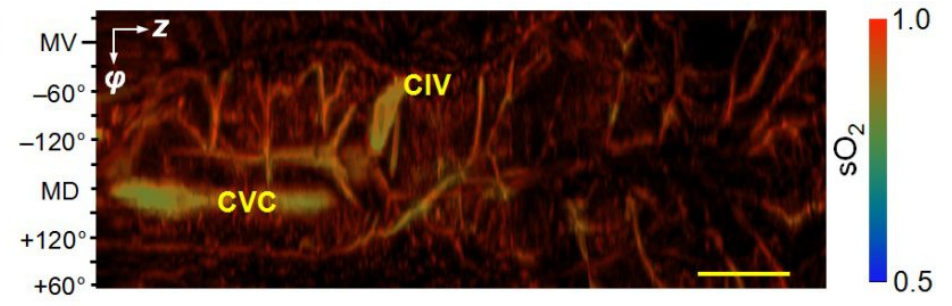

(b)

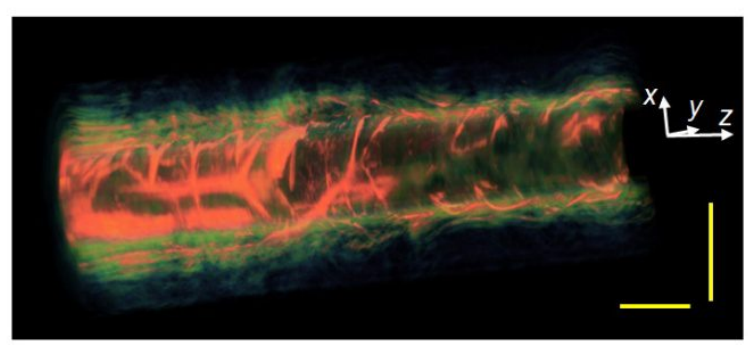

Figure 4. (a) A spectrally-processed PA-RMAP image acquired from the dorsal region of a rat colon ex vivo. The upper and lower parts of this image correspond to the left and right sides of the animal, and the middle section of the image is from the dorsal side of the animal. CVC, caudal vena cava; CIV, common iliac vein. The vertical $\varphi$-axis corresponds to the angular FOV covering $270^{\circ}$, and the horizontal $z$-axis corresponds to the pullback length of $8 \mathrm{~cm}$. The approximate mid-ventral (MV) position and angular measures from the MV are marked along the vertical $\varphi$-axis, where the positive and negative values correspond to the right and left sides of the animal, and MD denotes the mid-dorsal position. The scale bar represents $1 \mathrm{~cm}$ (only for the horizontal direction). (b) A threedimensionally-rendered PA structural image for the $523 \mathrm{~nm}$ data set shown in (a). The plotted image covers an $8 \mathrm{~cm}$ range with a 12 $\mathrm{mm}$ image diameter. The right-hand side $(+z)$ and $-y$ axis direction of the image correspond to the anus and the dorsal side of the animal, respectively. The horizontal and vertical scale bars represent $1 \mathrm{~cm}$ and $5 \mathrm{~mm}$, respectively.

We have demonstrated dual-wavelength PA endoscopic imaging by using two identical pulsed laser systems. Multiwavelength imaging based on a single laser system is theoretically possible; however, the required wavelength tuning time of our dye lasers is far too long. Additionally, laser systems that provide rapid tuning and sufficient pulse energies may not be commercially available. Long delays in wavelength tuning make it difficult to acquire co-registered dualwavelength PA images, and render measurements of some dynamic physiological parameters impossible. On the other hand, by utilizing the two identical laser systems, we could achieve rapid and co-registered dual-wavelength PA imaging. In order to couple light from two different laser sources into the endoscope, we had to develop light delivery optics as presented in Figure 2(a). The large physical distance between the laser sources complicated the design and implementation of the beam combiner [Fig. 2(a)], which could be simplified if the two laser systems were closer. 
The main advantage of dye laser-based wavelength tuning is the freedom in choosing from a wide spectrum of wavelengths for spectroscopic imaging. However, the cost of the employed laser systems is expensive $(\sim \$ 150 \mathrm{k}$ for each laser set) and they are bulky, which substantially restricts portability. In the future, however, the PAE light source could be comprised of a compact laser system, such as a Raman laser, which can provide dual laser wavelengths. We are currently working in this direction for clinical applications.

\section{CONCLUSIONS}

We developed peripheral optical systems for simultaneous dual-wavelength PA imaging that utilizes two identical pulsed-laser systems, and validated the developed systems through an ex vivo animal imaging experiment. The laser systems and implemented light delivery optics were able to provide adequate pulse energies and repetition rates for PAE at the scanning head of the endoscope $(\sim 0.3-0.6 \mathrm{~mJ}$ per pulse at a repetition rate of $\sim 1 \mathrm{kHz})$.

\section{ACKNOWLEDGEMENT}

We thank Prof. James Ballard for his attentive reading of the manuscript. This work was sponsored in part by National Institutes of Health grants R01 CA157277, R01 NS46214 (BRP), R01 EB000712, R01 EB008085, and U54 CA136398 (Network for Translational Research). L.W. has a financial interest in Microphotoacoustics, Inc. and Endra, Inc., which, however, did not support this work. J.-M.Y. was supported in part by a Korea Research Foundation Grant funded by the Korean Government (KRF-2007-357-C00039).

\section{REFERENCES}

1. Wang, L.V. Prospects of photoacoustic tomography. Med Phys 35, 5758-5767 (2008).

2. Yang, J.M., et al. Photoacoustic endoscopy. Opt Lett 34, 1591-1593 (2009).

3. Yang, J.M., et al. Volumetric photoacoustic endoscopy of internal organs: a phantom and in situ study. Proc. SPIE 7564, 75640D (2010).

4. Yang, J.M., et al. Volumetric photoacoustic endoscopy of upper gastrointestinal tract: ultrasonic transducer technology development Proc. SPIE 7899, 78990D (2011).

5. Beard, P. Biomedical photoacoustic imaging. Interface Focus 1, 602-631 (2011).

6. Yang, J.M., et al. Simultaneous functional photoacoustic and ultrasonic endoscopy of internal organs in vivo. Nat Med (2012).

7. Tearney, G.J., et al. In vivo endoscopic optical biopsy with optical coherence tomography. Science 276, 2037-2039 (1997).

8. Yun, S.H., et al. Comprehensive volumetric optical microscopy in vivo. Nat Med 12, 1429-1433 (2006).

9. Adler, D.C., et al. Three-dimensional endomicroscopy using optical coherence tomography. Nat Photon 1, 709-716 (2007).

10. Kiesslich, R., et al. Confocal laser endoscopy for diagnosing intraepithelial neoplasias and colorectal cancer in vivo. Gastroenterology 127, 706-713 (2004).

11. Qiu, L., et al. Multispectral scanning during endoscopy guides biopsy of dysplasia in Barrett's esophagus. Nat Med 16, 603-606, 601p following 606 (2010).

12. Terry, N.G., et al. Detection of dysplasia in Barrett's esophagus with in vivo depth-resolved nuclear morphology measurements. Gastroenterology 140, $42-50$ (2011).

13. Wang, L.V. Multiscale photoacoustic microscopy and computed tomography. Nat Photonics 3, 503-509 (2009).

14. Hu, S. \& Wang, L.V. Photoacoustic imaging and characterization of the microvasculature. J Biomed Opt 15, 011101 (2010).

15. Wang, B., et al. Intravascular photoacoustic imaging. IEEE Journal of Selected Topics in Quantum Electronics 16, $588-599(2010)$.

16. Wang, L.V. (ed.) Photoacoustic Imaging and Spectroscopy, (CRC, Boca Raton, 2009). 
17. Favazza, C.P., Cornelius, L.A. \& Wang, L.V. In vivo functional photoacoustic microscopy of cutaneous microvasculature in human skin. J Biomed Opt 16, 026004 (2011).

18. Chatni, M.R., et al. Functional photoacoustic microscopy of pH. J Biomed Opt 16, 100503 (2011).

19. Danielli, A., Favazza, C.P., Maslov, K. \& Wang, L.V. Single-wavelength functional photoacoustic microscopy in biological tissue. Opt Lett 36, 769-771 (2011).

20. Yao, J., Maslov, K.I., Shi, Y., Taber, L.A. \& Wang, L.V. In vivo photoacoustic imaging of transverse blood flow by using Doppler broadening of bandwidth. Opt Lett 35, 1419-1421 (2010).

21. Yao, J., Maslov, K.I., Zhang, Y., Xia, Y. \& Wang, L.V. Label-free oxygen-metabolic photoacoustic microscopy in vivo. J Biomed Opt 16, 076003 (2011).

22. Hu, S. \& Wang, L.V. Neurovascular photoacoustic tomography. Front Neuroenergetics 2, 10 (2010).

23. Maslov, K., Zhang, H.F., Hu, S. \& Wang, L.V. Optical-resolution photoacoustic microscopy for in vivo imaging of single capillaries. Opt Lett 33, 929-931 (2008).

24. Ku, G., Maslov, K., Li, L. \& Wang, L.V. Photoacoustic microscopy with 2-microm transverse resolution. J Biomed Opt 15, 021302 (2010).

25. Zhang, C., Maslov, K. \& Wang, L.V. Subwavelength-resolution label-free photoacoustic microscopy of optical absorption in vivo. Opt Lett 35, 3195-3197 (2010).

26. Li, C. \& Wang, L.V. Photoacoustic tomography and sensing in biomedicine. Phys Med Biol 54, R59-97 (2009).

27. Manohar, S., et al. Initial results of in vivo non-invasive cancer imaging in the human breast using near-infrared photoacoustics. Opt Express 15, 12277-12285 (2007).

28. Ermilov, S.A., et al. Laser optoacoustic imaging system for detection of breast cancer. J Biomed Opt 14, 024007 (2009).

29. Kruger, R.A., Lam, R.B., Reinecke, D.R., Del Rio, S.P. \& Doyle, R.P. Photoacoustic angiography of the breast. Med Phys 37, 6096-6100 (2010). 